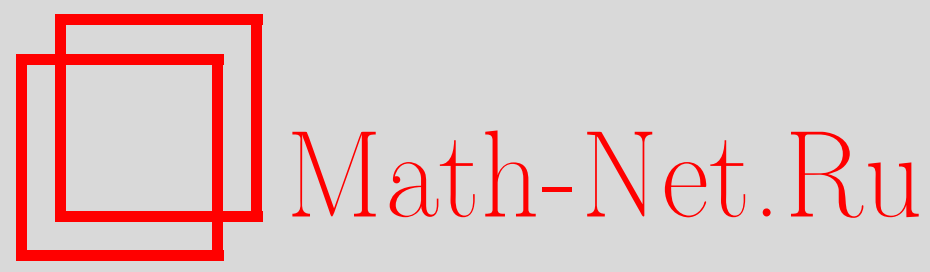

С. С. Габриелян, О проективных отображениях, Матем. заметки, 2002, том 72, выпуск 3, 323-329

DOI: https://doi.org/10.4213/mzm424

Использование Общероссийского математического портала Math-Net.Ru подразумевает, что вы прочитали и согласны с пользовательским соглашением http://www .mathnet.ru/rus/agreement

Параметры загрузки:

IP : 3.89 .185 .249

26 апреля 2023 г., 15:02:01

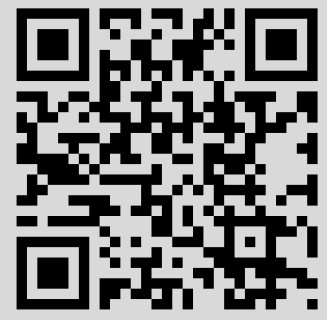




\title{
О ПРОЕКТИВНЫХ ОТОБРАЖЕНИЯХ
}

\author{
С. С. Габриелян
}

Пусть $X, Y$ - польские пространства и $\mathscr{B}_{k}$ есть $\sigma$-алгебра, порожденная проективным классом $L_{2 k+1}$. Отображение $f: X \mapsto Y$ называется $k$-проективньп, если $f^{-1}(E) \in \mathscr{B}_{k}$ для любого борелевского множества $E$. Основным результатом является следующая теорема: для любого $k$-проективного отображения $f: X \mapsto Y$ существует польское пространство $\widetilde{X}_{S}$, его плотное подмножество $X_{S} \in \mathscr{B}_{k}$ и два непрерывных отображения $f_{0}$ и $i$ из $\widetilde{X}_{S}$ в $Y$ такие, что

i) $\left.f_{0}\right|_{X_{S}}=\left.f \circ i\right|_{X_{S}}$;

ii) $\left.i\right|_{X_{S}}$ - биекция.

Библиография: 6 названий.

Идея "изменения топологии" является весьма полезной в многочисленных приложениях. Одним из наиболее существенных ее проявлений явилась теорема Куратовского (см. [1]), широко используемая в дескриптивной теории множеств (обобщение этой теоремы для борелевских $G$-пространств, где $G$-польская групша, см. [1]). Сформулируем классический результат Куратовского в следующем виде.

Теорема (Куратовский). Пусть $X$ - стандартное борелевское пространство, $Y$ - польское пространство и $f: X \rightarrow Y$ - борелевское отображсение. Тогда существуют польское пространство $\widetilde{X}_{S}$, его замкнутое подмножсество $X_{S}$ (значит, $X_{S}$ также польское), непрерывная биекция $i: X_{S} \rightarrow X$ и непрерывное отображсение $f_{0}: \widetilde{X}_{S} \rightarrow Y$ такие, что диаграмма

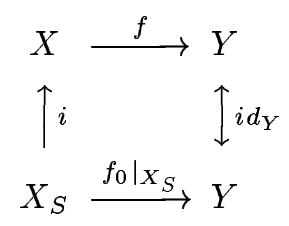

коммутативна.

Однако часто возникают не борелевские отображения, например, $A$-функции (см. [2, $\S 39, \mathrm{XI}])$. Поэтому естественно возникает вопрос об аналоге теоремы Куратовского, в частности, для $A$-функций. Получение этого аналога является главной целью данной статьи. В работе также рассматриваются некоторые применения полученных результатов. 
Под борелевским пространством $(X, \mathscr{B})$ понимается множество $X$ с выделенной на нем $\sigma$-алгеброй $\mathscr{B}$ его подмножеств, которые назьваются борелевскими. Если $X$ - топологическое пространство, а $\mathscr{B}$ порождена открытыми множествами топологии $\tau$ на $X$, то будем говорить, что $\mathscr{B}$ порождена $\tau$. Если на $X$ можно ввести топологию $\tau$ такую, что $\mathscr{B}$ порождена $\tau$, то будем говорить, что $\mathscr{B}$ допускает топологию. Через $\mathscr{B}(S)$ обозначим $\sigma$-алгебру, порожденную семейством множеств $S$. Если $\mathscr{B}=\mathscr{B}(S)$, где $S$ счетно, то говорят, что пространство обладает счетной базой. Борелевские пространства будем считать отделимыми (т.е. для каждой пары различных точек существует борелевское множество, содержащее ровно одну из этих точек). Стандартно вводятся борелевские отображения и изоморфизмы.

Пусть $L_{n}$ - семейство проективных множеств класса $n$ польского пространства $X$. Тогда $\mathscr{B}\left(L_{0}\right)$ состоит из “обычных" борелевских множеств, а $\mathscr{B}\left(L_{2 k-1}\right)=\mathscr{B}\left(L_{2 k}\right)$, $k \in \mathbb{N}$. Обозначим через $\mathscr{B}_{0}=\mathscr{B}\left(L_{0}\right), \mathscr{B}_{k}=\mathscr{B}\left(L_{2 k-1}\right), k \in \mathbb{N}$. Семейство $\mathscr{B}_{k}$ на $X$ будем обозначать через $\mathscr{B}_{k}(X)$. Легко показать, что $\mathscr{B}_{k} \subset L_{2 k+1} \cap L_{2 k+2}, k=1,2, \ldots$ Известно также, что $\mathscr{B}_{0} \varsubsetneqq \mathscr{B}_{1} \varsubsetneqq \mathscr{B}_{2} \varsubsetneqq \cdots$.

Непосредственно из $[2, \S 39, \mathrm{~V}$, теорема 4] вытекает утверждение: если $f$ - взаимно однозначное борелевское отображение, определенное на борелевском множестве $E$, то образ и прообраз множества из $\mathscr{B}_{k}$ также является множеством из $\mathscr{B}_{k}$.

Обобщением понятия $A$-функции является следующее

ОПРЕДЕЛЕНИЕ 1. Отображение $f$ из полного пространства $X$ в полное пространство $Y$ назьвается отображсением $k$-проективного класса, если $\forall E \in \mathscr{B}_{0}(Y) \Longrightarrow$ $f^{-1}(E) \in \mathscr{B}_{k}(X)$.

Основным результатом статьи является

Теорема 1. Пусть $X$ - стандартное борелевское пространство, $Y$ - польское пространство и $f: X \rightarrow Y$-отображсние $k$-проективного класса. Тогда существуют польское пространство $\widetilde{X}_{S}$, его плотное подмножество $X_{S} \in \mathscr{B}_{k}\left(\widetilde{X}_{S}\right)$, непрерывная биекиия $i: X_{S} \rightarrow Y$ и непрерывное отображение $f_{0}: \widetilde{X}_{S} \rightarrow Y$ такие, что диаграмма $(D)$ коммутативна.

Доказательство теоремы 1 делится на три этапа:

1) по исходному базису пространства $X$ и прообразам базиса пространства $Y$ вводится новая топология на $X\left(X\right.$ с новой топологией есть $\left.X_{S}\right)$;

2) строится пополнение $\widetilde{X}_{S}$ пространства $X_{S}$;

3) показывается, что $X_{S} \in \mathscr{B}_{k}\left(\widetilde{X}_{S}\right)$.

Первая и вторая части используют только наличие второй аксиомы счетности в пространствах $X$ и $Y$, поэтому пригодны для любых пространств со счетной базой.

Через $A^{(i)}$ будем обозначать $A$, если $i=1$, и $X \backslash A$, если $i=0$.

В дальнейшем мы будем рассматривать только пространства со счетной базой. Поэтому естественными аналогами понятий полноты и пополнения пространства, являются следующие определения (см. [3]).

ОПРЕДЕЛЕНИЕ 2. Отделимое пространство $X$ со счетной базой $S=\left\{A_{n}, n \in \mathbb{N}\right\}$ назьвается полным относительно $S$, если любой фильтр с базисом вида

$$
\mathscr{F}=\left\{E_{i_{1} \ldots i_{n}}=A_{1}^{\left(i_{1}\right)} \cap \cdots \cap A_{n}^{\left(i_{n}\right)}\right\}_{n=1}^{\infty}
$$

сходится (и состоит из единственной точки). 
ОПРЕДЕЛЕНИЕ 3. Отделимое пространство $\widetilde{X}$ с базой $\widetilde{S}=\left\{\widetilde{A}_{n}, n \in \mathbb{N}\right\}$ назьвается пополнением пространства $X$ относительно его базы $S=\left\{A_{n}, n \in \mathbb{N}\right\}$, если

1) пространство $\widetilde{X}$ полно относительно $\widetilde{S}$;

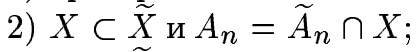

$3)$ если $\widetilde{\mathscr{F}}$ - базис фильтра вида $(1)$ в $\widetilde{X}$, то $\widetilde{\mathscr{F}} \cap X-$ базис фильтра в $X$.

Следующее предложение показывает, что класс отделимьх пространств со счетной базой совпадает с классом сепарабельных метрических пространств, а также устанавливает связь между полнотой пространства и полнотой относительно построенной метрики, что позволит дать простое доказательство первой части теоремы Рохлина (см. ниже).

ПРЕДЛОЖЕНИЕ 1. На отделимом пространстве $(X, \mathscr{B})$ со счетной базой $S=$ $\left\{A_{n}, n \in \mathbb{N}\right\}$ можно ввести метрику $\rho_{S}$ так, что

1) пространство $X$ является вполне несвязным сепарабельным метрическим пространством относительно $\rho_{S}$, все множества $A_{n}$ открыто-замкнуты и $\mathscr{B}$ порождена топологией;

2) пополнение пространства $X$ относительно базы $S$ существует, единственно и совпадает с пополнением $X$ по метрике $\rho_{S}$; кроме того, пространство $X$ полно относительно $S$ тогда и только тогда, когда $X$ полно относительно метрики $\rho_{S}$.

ДокАзАтЕльство. Первая часть доказана Ригером (см. [4, гл. XI, § 2, следствие]), существование и единственность пополнения доказаны Рохлиньп [3], оставшиеся утверждения доказьваются простой проверкой. Приведем лиш построение метрики (она понадобится при доказательстве предложения 3).

Пусть $D=\{0,1\}^{\mathbb{N}}$ - канторов дисконтинуум. Определим инъективное отображение из $X$ в $D$

$$
X \ni \bigcap_{n=1}^{\infty} A_{n}^{\left(i_{n}\right)}=x \mapsto\left(i_{1}, i_{2}, \ldots\right) \in D
$$

Из $D$ индуцируется метрика на $X$ :

$$
\rho_{S}(x, y)=\frac{1}{2^{\omega(x, y)}} \quad \text { при } x \neq y \quad \text { и } \quad \rho_{S}(x, x)=0 \quad \forall x \in X,
$$

где $\omega(x, y)$ - есть наименьшее натуральное число $n$ такое, что сушествуют числа $i_{1}, \ldots$ $\ldots, i_{n}$, для которых $x \in A_{1}^{\left(i_{1}\right)} \cap A_{2}^{\left(i_{2}\right)} \cap \cdots \cap A_{n}^{\left(i_{n}\right)}$, а $y \notin A_{1}^{\left(i_{1}\right)} \cap A_{2}^{\left(i_{2}\right)} \cap \cdots \cap A_{n}^{\left(i_{n}\right)}$. При этом система борелевских множеств

$$
\mathscr{N}=\left\{E_{i_{1} i_{2} \ldots i_{n}}=A_{1}^{\left(i_{1}\right)} \cap A_{2}^{\left(i_{2}\right)} \cap \cdots \cap A_{n}^{\left(i_{n}\right)}, i_{j}=0,1, j=1, \ldots, n ; n \in \mathbb{N}\right\}
$$

образует базу топологии.

СлеДСТВИЕ 1. Пусть $X$ - сепарабельное метрическое пространство $u\left\{B_{n}\right\}-$ база топологии. Тогда система $\mathcal{N}$, построенная по базе, представляет собой совериенное разбиение, состоящее из множеств типа $G_{\delta}$.

(Отметим также, что существование совершенных разбиений в таких пространствах доказано в [5].)

Первьй этап опирается на следующий известный результат. 
ПРЕДЛОЖЕНИЕ 2. Пусть $\left(X, \mathscr{B}_{X}\right) u\left(Y, \mathscr{B}_{Y}\right)$ - отделимые борелевские пространства со счетной базой и $f_{1}(x), f_{2}(x), \ldots$ - последовательность борелевских отображсений из $X$ в $Y$. Тогда на $X$ и $Y$ можсно ввести топологии $\tau_{X}^{*} u \tau_{Y}^{*}$ так, чтобы все $f_{k}(x)$ стали непрерывными (если $\mathscr{B}_{X}$ и $\mathscr{B}_{Y}$ порожсдены топологиями $\tau_{X}$ и $\tau_{Y}$, то на $X$ можнно ввести топологию $\tau_{X}^{*}$, возможнно более сильную, чем $\tau_{X}$, так, чтобы все $f_{k}(x)$ стали непрерывными). При этом если все $f_{k}(x)-$ борелевские изоморфизмы, то можнно ввести топологии (или топологию, когда $X=Y)$, возможнно более сильные, чем исходные, так, что все $f_{k}(x)$ станут гомеоморфизмами.

ДоказАтЕльство. Пусть $S=\left\{A_{j}, j \in \mathbb{N}\right\}-$ базапространства $Y$ и $\mathscr{N}=\left\{E_{i_{1} \ldots i_{n}}\right\}-$ система множеств, построенная по $S$ в предложении 1. Тогда эта система определяет на $Y$ базу $\left\{A_{j}^{\prime}, j \in \mathbb{N}\right\}$ топологии $\tau_{Y}^{*}$ сепарабельного метрического пространства.

Построим, как в предложении 1 , топологию $\tau_{X}^{*}$ на $X$ по системе множеств

$$
\left\{B_{i}, f_{k}^{-1}\left(A_{j}^{\prime}\right) ; i, j, k \in \mathbb{N}\right\}
$$

где $\left\{B_{i}\right\}$ - база пространства (топологии $\tau_{X}$ ) на $X$, а $A_{j}^{\prime}$ - либо построенная вьше система, либо база топологии $\tau_{Y}$ на $Y$, если $Y$ является топологическим пространством. Тогда

1) топология $\tau_{X}^{*}$, вообще говоря, сильнее исходной, если таковая была;

2) все отображения $f_{k}$ непрерьвны, так как по предложению $1 f_{k}^{-1}\left(A_{j}^{\prime}\right) \in \tau_{X}^{*}$.

Оставшаяся часть доказьвается аналогично.

Третий этап содержится в следующем предложении.

ПРЕДЛОЖЕНИЕ 3. Пусть $S=\left\{A_{n}, n \in \mathbb{N}\right\} \subset \mathscr{B}_{k}$ u coдержит базу топологии, а $X_{S}$ - метрическое пространство, построенное по базе $S$. Тогда $X_{S}$ есть мнохсество из $\mathscr{B}_{k}$ в своем пополнении $\widetilde{X}_{S}$. При этом непрерывное тохсдественное отображение $i$ из $X_{S}$ на $X$ осуществляет изоморфизм пространств $\left(X_{S}, \mathscr{B}_{0}\left(\widetilde{X}_{S}\right) \cap X_{S}\right) u(X, \mathscr{B}(S))$.

ДокАЗАТЕЛьСТво разобьем на пять шагов.

1) Пусть $X_{0}=X$. Положим

$$
X_{n}=\sqcup \mathrm{Cl}\left(A_{1}^{\left(i_{1}\right)} \cap \cdots \cap A_{n}^{\left(i_{n}\right)}\right)
$$

- дизъюнктное объединение замыканий в $X$ множеств $A_{1}^{\left(i_{1}\right)} \cap \cdots \cap A_{n}^{\left(i_{n}\right)}$ с индуцированной из $X$ метрикой. Тогда $X_{n}$ - полное сепарабельное метрическое пространство. Определим непрерьвную проекцию $\pi_{n+1, n}$ из $X_{n+1}$ в $X_{n}$ следующим образом:

$$
\text { если } x \in \mathrm{Cl}\left(A_{1}^{\left(i_{1}\right)} \cap \cdots \cap A_{n}^{\left(i_{n}\right)} \cap A_{n+1}^{\left(i_{n+1}\right)}\right) \text {, то } \pi_{n+1, n}(x)=x \in \mathrm{Cl}\left(A_{1}^{\left(i_{1}\right)} \cap \cdots \cap A_{n}^{\left(i_{n}\right)}\right),
$$
и пусть $\pi_{n, l}=\pi_{n, n-1} \circ \cdots \circ \pi_{l+1, l}, n>l$. Тогда очевидно, что система $\left(X_{n}, \pi_{n, l}\right)$ является проективной. Обозначим через $Y_{0}$ проективньй предел этой системы. Тогда $Y_{0}$ - полное сепарабельное метрическое пространство, которое является замкнутым подпространством прямого произведения $Y=\prod_{n=0}^{\infty} X_{n}$ пространств $X_{n}$.

2) Построим отображение $p_{n}$ из $X=X_{0}$ в $X_{n}$, полагая $p_{0}(x)=x$, и если $x \in A_{1}^{\left(i_{1}\right)} \cap \cdots \cap A_{n}^{\left(i_{n}\right)}$, то $p_{n}(x)=x \in \operatorname{Cl}\left(A_{1}^{\left(i_{1}\right)} \cap \cdots \cap A_{n}^{\left(i_{n}\right)}\right)$. 
Тогда $p_{n}$ инъективно и удовлетворяет условию:

образы и прообразы множеств из $\mathscr{B}_{k}$ принадлежат $\mathscr{B}_{k}$.

Действительно, $E \in \mathscr{B}_{k}(X)$ тогда и только тогда, когда

$$
E \cap\left(A_{1}^{\left(i_{1}\right)} \cap \cdots \cap A_{n}^{\left(i_{n}\right)}\right) \in \mathscr{B}_{k}(X),
$$

что равносильно тому, что

$$
E \cap\left(A_{1}^{\left(i_{1}\right)} \cap \cdots \cap A_{n}^{\left(i_{n}\right)}\right) \in \mathscr{B}_{k}\left(\mathrm{Cl}\left(A_{1}^{\left(i_{1}\right)} \cap \cdots \cap A_{n}^{\left(i_{n}\right)}\right)\right)
$$

или $E \in \mathscr{B}_{k}\left(X_{n}\right)$. В частности, $p_{n}(X) \in \mathscr{B}_{k}\left(X_{n}\right)$.

Обратно, если $E \in \mathscr{B}_{k}\left(X_{n}\right)$, то $E \cap p_{n}(X) \in \mathscr{B}_{k}\left(X_{n}\right)$. И, как доказано вьше, $p_{n}^{-1}(E)=p_{n}^{-1}\left(E \cap p_{n}(X)\right) \in \mathscr{B}_{k}(X)$.

При этом по построению верно равенство

$$
\pi_{n+1, n}\left(p_{n+1}(x)\right)=p_{n}(x) .
$$

3) Пусть $i$ - непрерывное тождественное отображение из $X_{S}$ в $X$. Тогда $p_{n} \circ i$ непрерьвно.

Действительно, пусть $x \in X_{S}$ и $V$-окрестность точки $p_{n}(i(x)) \in \mathrm{Cl}\left(A_{1}^{\left(i_{1}\right)} \cap \cdots \cap A_{n}^{\left(i_{n}\right)}\right)$ $\subset X_{n}$. Тогда существуют открытое подмножество $U$ в $X$ и элемент $A_{l} \in S$ такие, что $i(x) \in A_{l} \subset U$ и $U \cap \operatorname{Cl}\left(A_{1}^{\left(i_{1}\right)} \cap \cdots \cap A_{n}^{\left(i_{n}\right)}\right)=V$. Тогда $i(x) \in A_{1}^{\left(i_{1}\right)} \cap \cdots \cap A_{n}^{\left(i_{n}\right)} \cap A_{l}=W$. Так как $W$ открыто в $X_{S}$ и $p_{n}(i(y))=y \in \operatorname{Cl}\left(A_{1}^{\left(i_{1}\right)} \cap \cdots \cap A_{n}^{\left(i_{n}\right)}\right) \cap A_{l} \subset V \forall y \in W$, то $p_{n} \circ i$ непрерывно.

4) Определим инъективное отображение $p$ из $X$ в $Y$, полагая

$$
p(x)=\left(p_{0}(x), p_{1}(x), \ldots\right) .
$$

Из $(*)$ следует, что $p(X) \subset Y_{0}$. Покажем, что

если $E \in \mathscr{B}_{0}(X)$, то $p(E) \in \mathscr{B}_{k}\left(Y_{0}\right)$;

в частности, $p(X) \in \mathscr{B}_{k}\left(Y_{0}\right)$. Отображение $p$ можно представить в виде композиции двух отображений $p=f \circ g$, где $g$ - тождественный гомеоморфизм $X$ на диагональ в $Z=\prod_{n=0}^{\infty} X$, a $f$ - отображение из $Z$ в $Y$, определенное следуюшим образом:

$$
f\left(x_{0}, x_{1}, \ldots\right)=\left(p_{0}\left(x_{0}\right), p_{1}\left(x_{1}\right), \ldots\right) .
$$

Так как $Y_{0}$ замкнуто в $Y$, то достаточно доказать, что если $E \in \mathscr{B}_{0}(Z)$, то $f(E) \in$ $\mathscr{B}_{k}(Y)$. Но $f$ инъективно, поэтому можно считать, что $E=A_{0} \times A_{1} \times \ldots$, где $A_{i} \in$ $\mathscr{B}_{0}\left(X_{i}\right)$. Так как

$$
Y \backslash f(E)=\bigcup_{n=0}^{\infty} X_{0} \times \cdots \times X_{n-1} \times\left(X_{n} \backslash p_{n}\left(A_{n}\right)\right) \times X_{n+1} \times \cdots
$$

и по части 2) $X_{n} \backslash p_{n}\left(A_{n}\right) \in \mathscr{B}_{k}\left(X_{n}\right)$, то $f(E) \in \mathscr{B}_{k}$.

$5)$ Покажем, что взаимно однозначное отображение $h=p \circ i$ из $X_{S}$ в $Y_{0}$ есть гомеоморфизм $X_{S}$ на $h\left(X_{S}\right)$. 
Непрерывность $h$ вытекает из пункта 3). Пусть $x, y \in X_{S}$ и $\rho_{S}(x, y)=1 / 2^{m+1}$, т.е. $x, y \in A_{1}^{\left(i_{1}\right)} \cap \cdots \cap A_{m}^{\left(i_{m}\right)}$, a $x \in A_{1}^{\left(i_{1}\right)} \cap \cdots \cap A_{m}^{\left(i_{m}\right)} \cap A_{m+1} \not \supset y$. Тогда $\rho_{X_{n}}\left(p_{n}(i(x))\right.$, $\left.p_{n}(i(y))\right)=\rho(x, y)$ при $n \leqslant m$ и $\rho_{X_{n}}\left(p_{n}(i(x)), p_{n}(i(y))\right)=1$ при $n>m$, a

$\rho_{Y}(h(x), h(y))=\sum_{n=0}^{\infty} \frac{1}{2^{n}} \rho_{X_{n}}\left(p_{n}(i(x)), p_{n}(i(y))\right)=\rho(x, y) \cdot\left(2-\frac{1}{2^{m}}\right)+\frac{1}{2^{m}} \geqslant \rho_{S}(x, y)$,

поэтому обратное отображение также непрерывно и $h$ - гомеоморфизм.

По теореме Лаврентьева $h$ можно продолжить до гомеоморфизмов $G_{\delta}$ множеств в $\widetilde{X}_{S}$ и $Y_{0}$. Так как по пункту 4) $h\left(X_{S}\right) \in \mathscr{B}_{k}\left(Y_{0}\right)$, то $X_{S}=h^{-1}\left(h\left(X_{S}\right)\right) \in \mathscr{B}_{k}\left(\widetilde{X}_{S}\right)$. Последнее утверждение вытекает из предложения 1.

ДоКАЗАТЕЛЬСТво ТЕОРЕМЫ 1. По теореме Куратовского можно, не ограничивая общности, считать, что $X$ польское. Пусть $S=\left\{B_{i}, f^{-1}\left(A_{j}\right) ; i, j \in \mathbb{N}\right\}$, где $\left\{A_{j}\right\}-$ база топологии на $Y$, a $\left\{B_{i}\right\}$ - база топологии на $X$. Положим $E=X_{S}$ и $X_{0}=\widetilde{X}_{S}$-пополнение $X_{S}$. Как и в предложении 1 , тождественная биекция $i$ множества $E$ на $X$ и $f_{0}=f \circ i$ непрерьвны. По предложению $3 E \in \mathscr{B}_{k}\left(X_{0}\right)$. Продолжив $f_{0}$ и $i$ на $G_{\delta}$-множество и снова воспользовавшись теоремой Куратовского, получим требуемое.

Отметим, что аналогичную теорему можно сформулировать и для последовательности отображений $k$-проективного класса.

\section{Применения}

1. Используя предложения 1-3 и свойство совершенного ядра, докажем теорему.

ТЕОремА 2. б-алгебра, порожденная аналитическими множествами, имеет несчетный вес.

ДоказАтельство. Допустим противное: $S=\left\{A_{n}, n \in \mathbb{N}\right\} \subset \mathscr{B}_{1}$ и $\mathscr{B}(S)=\mathscr{B}_{1}$. Не ограничивая общности, можно считать, что база топологии $X$ содержится в $S$. Пусть $X_{S}$ - пространство $X$ с метрикой $\rho_{S}, \widetilde{X}_{S}$ - пополнение $X_{S}$ и $i$ - непрерьвное тождественное отображение из $X_{S}$ на $X$ (см. предложение 1$)$. Тогда по предложению $3 \mathscr{B}_{1}=$ $i\left(\mathscr{B}_{0} \cap X_{S}\right)$ и $X_{S}-$ множество класса $\mathscr{B}_{1}$ в $\widetilde{X}_{S}$. Так как $X$ имеет мошность континуума, то в $X_{S}$ имеется борелевское (в $\left.\widetilde{X}_{S}\right)$ подмножество $E$ мошности континуум [2, $339, \mathrm{II}$, следствие 3]. Пусть $A$ - аналитическое неборелевское подмножество в $E$ (а, значит, и в $\left.\widetilde{X}_{S}\right)$. Тогда $A \notin \mathscr{B}_{0} \cap X_{S}$, но $i(A) \in \mathscr{B}_{1}$. Противоречие.

Отметим, что, используя идеи предложений 1-3, можно прийти, фактически, к более обшим результатам статьи [6].

2. Пространства Лебега. Из предложений 1-3 легко доказать первую часть теоремы Рохлина (основные понятия и определения см. [3]), а именно:

ТЕОрема 3. 1. Класс сепарабельных пространств $\left(X, \Omega_{\mu}, \mu\right)$ совпадает с классом сепарабельных метрических пространств с заданными борелевскими мерами.

2. Для любого пространства Лебега $\left(X, \Omega_{\mu}, \mu\right)$ существуют полное сепарабельное метрическое пространство $\widetilde{X}$ и заданная на нем мера $\widetilde{\mu}$ такие, что $X$ мохно рассматривать как $\widetilde{\mu}$-измеримое подмножсество в $\widetilde{X}$ полной меры, а $\mu$-как ограничение меры $\widetilde{\mu}$ на $X$. 
ДокАЗАтЕльство. 1. Пусть $S$ - база пространства $X$. Построим по $S$ метрику $\rho_{S}$. Тогда $X$ станет сепарабельным метрическим пространством и мера $\mu$ определена на всех борелевских множествах $\mathscr{B}$. Обозначим ограничение $\mu$ на $\mathscr{B}$ через $\mu_{0}$. Через $\widetilde{\mu}$ обозначим продолжение меры $\mu_{0}$ на все $\mu_{0}$-измеримые множества. Тогда $\widetilde{\mu}-$ полная борелевская мера на $X$, а сепарабельность пространства как раз означает, что $\widetilde{\mu}=\mu$.

2. Немедленно вытекает из утверждения 1) предложения 1 и определения пространства Лебега.

3. В заключение продемонстрируем применение предложения 2 для доказательства теоремы Лузина в случае борелевского отображения.

ТЕОрема ЛУЗИнА. Пусть $X$ и $Y$ - сепарабельные метрические пространства, $\mu$-вероятностная регулярная мера на $X$ u $f(x)$ - борелевское отобрахсение из $X$ в $Y$. Тогда для любого $\varepsilon>0$ существует компактное множество $K_{\varepsilon} \subset X$ такое, что

1) $\mu\left(K_{\varepsilon}\right)>1-\varepsilon$;

2) сужение $f$ на $K_{\varepsilon}$ непрерывно.

ДокАЗАтЕльство. Заменяя $X$ на компакт меры больше, чем $1-\varepsilon / 2$, можно считать, что $X$ полно. Пусть $\tau_{X}$ - топология на $X$, возможно более сильная, чем исходная, относительно которой отображение $f$ непрерьвно. Пространство $X$ с новой топологией обозначим через $X_{0}$, функцию $f$ в новой топологии - через $f_{0}$, а тождественное непрерьвное отображение из $X_{0}$ в $X$ - через $i$. Пусть $\bar{X}_{0}$ - пополнение пространства $X_{0}$. Так как $X$ полно и $i$ - борелевский изоморфизм между $X$ и $X_{0}$, то $i^{-1}(X)=X_{0}$ - борелевское подмножество в $\bar{X}_{0}[2, \S 39, \mathrm{~V}$, теорема 1$]$.

Определим борелевскую меру $\bar{\mu}$ в $\bar{X}_{0}: \bar{\mu}(E)=\mu\left(i\left(E \cap X_{0}\right)\right)$, где $E-$ борелевское подмножество $\bar{X}_{0}$. Тогда $\bar{\mu}$ сосредоточена на $X_{0}$. Так как $\bar{X}_{0}$ полное, то $\bar{\mu}$ регулярна. Значит, для любого $\varepsilon>0$ существует компакт $\bar{K}_{\varepsilon} \subset X_{0}$ такой, что $\bar{\mu}\left(\bar{K}_{\varepsilon}\right)>1-\varepsilon$. Пусть $K_{\varepsilon}=i\left(\bar{K}_{\varepsilon}\right)$ - компакт в $X$. Покажем, что $K_{\varepsilon}$ искомый. По построению $\mu\left(K_{\varepsilon}\right)=$ $\bar{\mu}\left(\bar{K}_{\varepsilon}\right)>1-\varepsilon$. Так как $i$ непрерывно и взаимно однозначно, то $\left.i\right|_{K}-$ гомеоморфизм. Значит, $\left.f\right|_{K_{\varepsilon}}=f_{0} \circ\left(\left.i^{-1}\right|_{K_{\varepsilon}}\right)$ непрерьвно. Теорема доказана.

\section{СПИСОК ЦИТИРОВАННОЙ ЛИТЕРАТУРЫ}

[1] Kechris A.S. Descriptive dynamics // London Math. Soc. Lecture Notes. 2000. V. 277. P. 231-258.

[2] Куратовский К. Топология. Т. 1. М.: Мир, 1966.

[3] Рохлин В.А. Об основных понятиях теории меры // Матем. сб. 1949. Т. 67. №1. С. 107-150.

[4] Биркгоф Г. Теория решеток. М.: Наука, 1984.

[5] Morse A. P. Perfect blankets // Trans. Amer. Math. Soc. 1947. V. 61. № 1. P. 418-442.

[6] Rao B. V. Non-existence of certain Borel structures // Fund. Math. 1970. V. 69. P. 241-242.

Харьковский государственный политехнический университет, Украина

Поступило

E-mail: gabrss@kpi.kharkov.ua

10.04 .2001 